\title{
Novel self-consistent linear theory of a gyrotron oscillator and experimental validation
}

\author{
J. Genoud, T.M. Tran, S. Alberti, F. Braunmueller, J.-Ph. Hogge, M.Q. Tran, \\ Centre de Recherches en Physique des Plasmas, Ecole Polytechnique Fédérale de Lausanne (EPFL), \\ Station13, CH-1015, Lausanne, Switzerland,
}

\begin{abstract}
Based on a linearized set of self-consistent equations describing the time-dependent self-consistent waveparticle interaction in a gyrotron oscillator, a spectral approach is used for determining the spectrum of stable and unstable self-consistent eigenmodes. The model is an extension of a recently developed model based on a moment approach in [1] and retains all the relevant system inhomogeneities such as cavity-wall and magnetic field profiles. It is shown that this model predicts a new set of eigenmodes in addition to the set of eigenmodes issued from the cold-cavity modes. The model, the numerical implementation together with results for a real gyrotron cavity will be presented, discussed and compared to experimental results.
\end{abstract}

\section{INTRODUCTION}

$\mathrm{T}_{\mathrm{i}}$ HE linear theory of the electron cyclotron maser instability has been treated in the past in a large variety of models each one having a specific domain of applicability depending on the underlying assumptions upon which the model has been derived. A fairly extensive reference list for these models can be found in [2,3]. For the case of an infinitely long homogeneous waveguide supporting any given TE or TM modes, the most basic model, allows to derive the wave dispersion relation, by solving the self-consistent linearized Maxwell-Vlasov set of equations and assuming an equilibrium distribution function corresponding to a spatially homogeneous weakly relativistic magnetized electron beam [2]. This assumption yields to a dispersion relation in the $\left(\omega, \mathrm{k}_{\mathrm{z}}\right)$ space where $\omega$ is the complex angular frequency and $\mathrm{k}_{\mathrm{z}}$ is the real longitudinal wave number $\left(\mathrm{k}_{\mathrm{z}}\right.$ being parallel to the waveguide axis). In the case of a gyrotron oscillator, the cylindrical cavity supporting any given $\mathrm{TE}_{\mathrm{mp}}$ is finite in length and has a weakly inhomogeneous cross-section. The most common linear theory for a finite length nonhomogeneous cavity is non-selfconsistent [2] since a longitudinal eigenmode of the empty cavity mode (cold cavity, i.e. without electron beam) is considered for computing the self-excitation conditions expressed in terms of a starting current. A fully self-consistent approach consists in solving the linearized wave-particle system of equations, which, when solved in time domain gives the starting current (inversely proportional to the linear growth rate) of the most unstable mode $[1,4]$. In the novel approach presented in this paper, the linearized system of equations of [1] is generalized and is solved in the frequency domain (spectral approach), therefore allowing to study not only the most unstable mode, but a large number of stable and unstable modes, thus giving a significant more physical insight to the linear dynamics of the electron cyclotron maser instability.

\section{THEORETICAL MODEL}

The novel model described in this paper is an extension of a recent work by Braunmueller et al. [1]. In [1] the linearized set of time-dependent equations describing the selfconsistent dynamics of a single TE-mode, excited in a gyrotron oscillator, has been reduced, using the moment approach, to a system of PDE's describing the evolution of 3 complex fields all depending on the axial coordinate and time. The interaction space considers realistic cavity-wall profiles up to the uptaper following the main interaction region, takes into account the inhomogeneous magnetic field profile and self-consistently includes the cavity wall-losses. In [1], the system of equations has been solved in time domain allowing the determination of the self-consistent starting current conditions associated to the excitation of the most linearly unstable mode. In the reduced system of PDE's derived in [1] the commonly used assumption considering a constant wave-field envelope during the electron transit time is adopted.

In this paper, this last assumption is relaxed and the system of PDE's is generalized to the case where the wave-field envelope can vary during the electron transit time. Transforming this generalized set of equations in frequency domain yields to an ODE coupled system of homogeneous equations, shown below, which is solved as an eigenvalue problem.

$$
\left\{\begin{aligned}
\beta_{z} \frac{\mathrm{d} \pi_{1}}{\mathrm{~d} \hat{z}}-\left(i \tilde{\Delta}_{0}+i \tilde{C}_{1}+\beta_{z} \delta\right) \pi_{1}-i \tilde{C}_{1} \pi_{2}+\tilde{C}_{2} C_{0} F & =i \Omega \pi_{1} \\
\beta_{z} \frac{\mathrm{d} \pi_{2}}{\mathrm{~d} \hat{z}}-\left(i \tilde{\Delta}_{0}-i \tilde{C}_{1}+\beta_{z} \delta\right) \pi_{2}+i \tilde{C}_{1} \pi_{1} & =i \Omega \pi_{2} \\
\frac{\mathrm{d}^{2} F}{\mathrm{~d} \hat{z}^{2}}+\kappa_{\| 0}^{2} F-i C_{3} C_{0} \pi_{1} & =-2 \Omega F
\end{aligned}\right.
$$

In the system of equations, the fields $\pi_{1}(\Omega, z)$ and $\pi_{2}(\Omega, z)$ correspond to moments of the linearized electron perpendicular momenta and $\mathrm{F}(\Omega, \mathrm{z})$ is the rf-field envelope. The definitions of all quantities in the system of equations can be found in [1]. The system of equations supplemented with the appropriate boundary conditions (see [1]) defines an eigenvalue problem with $\Omega$ being the complex eigenvalue.

The spectrum of eigenvalues (spectral approach) describes the possible linearly unstable and stable oscillating modes. This novel method allows to study a large set of eigenmodes in a coupled spatially inhomogeneous system and therefore gives more physical insight on the linear phase taking place in a gyrotron oscillator. The novel model, the numerical implementation in the TWANGlinspec code and results for real gyrotron cavities will be presented and discussed with respect to experimental results obtained with a THzgyrotron described in $[5,6]$ as well as for high-power gyrotrons. 


\section{RESULTS}

Two examples of results obtained with the TWANGlinspec code are given here. First for a gyrotron cavity described in [5], and considering relevant system parameters for this experiment, a subset of calculated eigenvalues and corresponding eigenvectors are shown in Fig.1. The main point to be highlighted is that the spectral approach shows a new set of stable eigenvalues with an equidistant frequency spacing. A subset of the "singular" modes have their frequency below the cavity cutoff. For the "singular" mode highlighted by the red-diamond in Fig. $1 \mathrm{c}$ ), the amplitude of the corresponding eigenvector has its maximum in the small angle $\left(\theta=1^{0}\right)$ uptaper following the constant radius cavity section. The frequency equidistancy of the "singular" modes together with their frequency below the cavity cutoff are reminiscent of the properties of the side-bands experimentally observed in $[5,6]$. Moreover, the parametric dependence of the side-bands of [5,6], is in good agreement with the parametric dependence of the "singular" modes predicted by the novel model.
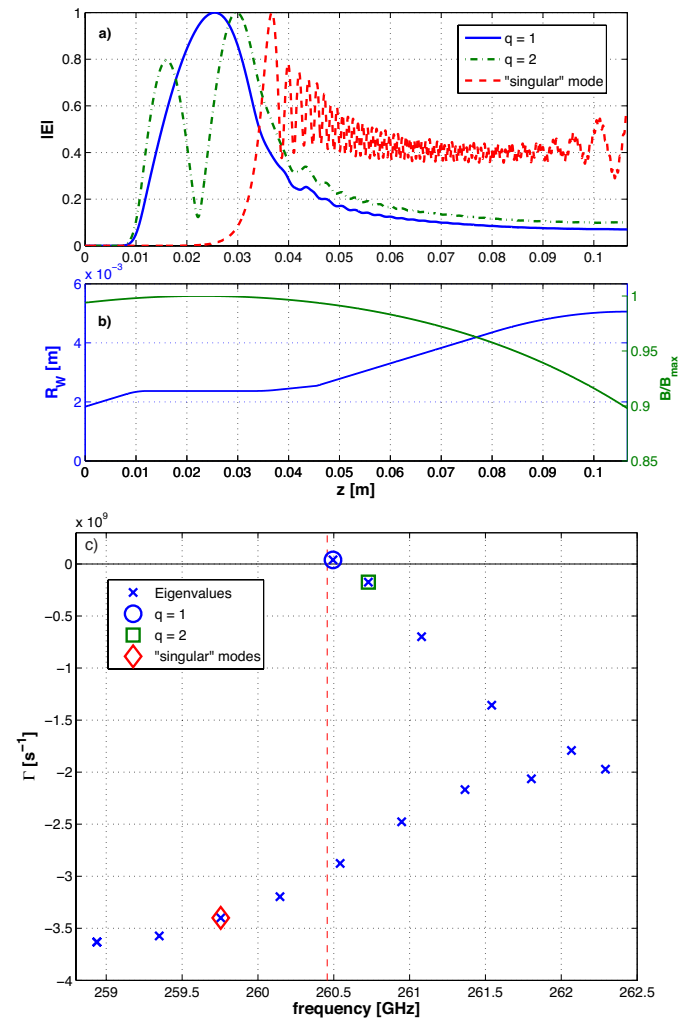

Figure 1. Subplot b): cavity geometry and magnetic field profile. Subplot c): complex eigenvalues calculated for a given set of system parameters. The beam current is $10 \mathrm{~mA}$. The most unstable eigenvalue $(\Gamma>0)$ corresponds to the $q=1$ longitudinal mode and is highlighted with the blue circle. Notice the new set of stable eigenvalues $(\Gamma<0)$ with an equidistant frequency spacing. The dashed vertical red-line indicates the cutoff frequency in the constant radius cavity section. Subplot a): eigenvectors for the three highlighted eigenvalues shown in subplot c). The blue and green curves correspond to the $q=1$ and $q=2$ profiles which can be identified from the corresponding cold-cavity eigenvectors. The novel "singular mode" is highlighted by the red diamond in c) with the amplitude of corresponding complex eigenvector shown in a) with the red dashed line.

In Figure 2, is shown the comparison of the results of the novel model with experimental results.

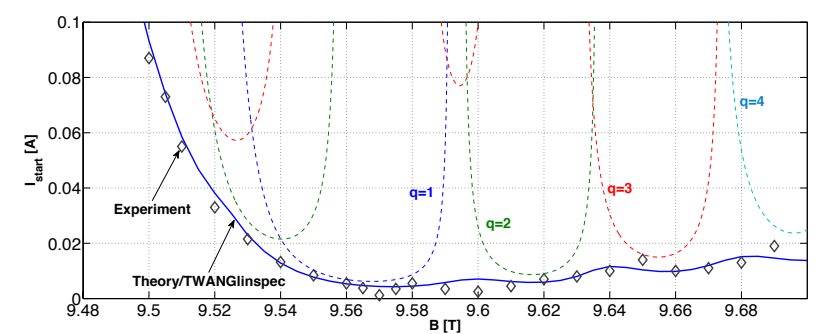

Figure 2. Comparison theory-experiment of the starting current versus magnetic field curve. The continuous bluecurve is the calculated minimum starting current, whereas the diamonds are measured starting currents for the THzgyrotron described in [5,6]. The dashed curves correspond to the minimum starting currents based on cold cavity profiles described by the lowest order longitudinal eigenmodes $q=1-4$.

The agreement between the theory and experiment is excellent. In this same figure, the starting currents based on a fixed field (cold cavity) calculation are also shown and clearly show the limitations of the fixed field approach.

\section{CONCLUSION}

The novel self-consistent model based on a spectral approach for studying the linear dynamics of the electron cyclotron maser instability allows to study a large set of eigenmodes in a coupled spatially inhomogeneous system and therefore gives more physical insight on the linear dynamics taking place in a gyrotron oscillator. The results obtained with TWANGlinspec for real gyrotron cavities show an excellent agreement with experimental results given in $[5,6]$. The parametric dependencies of these modes are in good agreements with the experimentally observed side-bands observed in $[5,6]$.

This model will also be used for studying the excitation of spurious instabilities in beam-ducts [4,7] for systems in which the boundary condition on the smooth beam-duct wall is described by an impedance boundary condition.

\section{ACKNOWLEDGMENTS.}

Work partially supported by by EFDA under Grant WP13-DAS-HCD-EC and by Fusion for Energy under Grant F4E-GRT-432 and within the European Gyrotron Consortium (EGYC). The views and opinions expressed herein do not necessarily reflect those of the European Commission. EGYC is a collaboration of CRPP, Switzerland; KIT, Germany; HELLAS, Greece; IFP-CNR, Italy.

\section{REFERENCES}

[1] F. Braunmueller et al., Phys. Plasmas, 21, 043105 (2014)

[2] K.R Chu, Rev. Mod. Phys., 76, 489 (2004)

[3] G. Nusinovich, Introduction to the Physics of Gyrotrons, John Hopkins (2004)

[4] J. Yu et al., IEEE Trans. Plasma Sci., 38 (6), 1193 (2010)

[5] S. Alberti et al.,. Phys. Plasmas, 19, 123102 (2012)

[6] S. Alberti et al., Phys. Rev. Lett., 111, 205101 (2013).

[7] L.Thorndahl et al., Proc. 36th Int. Conf. on IRMMW-THz, Houston (TX), 2011 